\title{
A Local Modified U-net Architecture for Image Denoising
}

\author{
Latha $\mathrm{H} \mathrm{N}^{1,2} \mid$ Rajiv R Sahay ${ }^{3}$ \\ ${ }^{1}$ Department of Computer Science, IIT, KGP, West Bengal, India \\ ${ }^{2}$ Department of E \& C, BMS College of Engineering , Bangalore, Karnataka, India \\ ${ }^{3}$ Department of E \& E, IIT, KGP, West Bengal, India
}

\section{To Cite this Article}

Latha H N and Rajiv R Sahay, "A Local Modified U-net Architecture for Image Denoising ", International Journal for Modern Trends in Science and Technology, 6(8S): 140-144, 2020.

\section{Article Info}

Received on 16-July-2020, Revised on 15-August-2020, Accepted on 25-August-2020, Published on 28-August-2020.

\section{ABSTRACT}

Image denoising is long well-studied issues in image processing, reconstruction and computer vision field, for a variety of image modelling problems. In this proposed work, an extension to traditional deep CNNs, Global skip connection, Normalization, Local pixels statistics, are included to obtain faster training and testing convergence of the model called Local Modified U-net (LM U-net). High frequency information of the pixels lost during decoding process is restored at the encoder end by processing local neighbor pixels. Results shown over60,000 training images, global skip connections providedgood improvements in feature learning for image restoration and denoising. The proposed LM U-net can also be effectively utilized for many other image restoration tasks similar to image super resolution, image deblurring

KEYWORDS: U-net 1, Image Segmentation 2, DCNN 3, LM U-net 4, decoder and encoder 5.

\section{INTRODUCTION}

Many imaging devices like digital cameras, smart phone and video cameras have invaded in to our lives. With these devices we can take more sophisticated and clear images/photographs than earlier. However, there are chances that the photos taken by such images may have more noise associated with them [1]-[3]. Noise may be generated while capturing a photograph, during transmission or while restoring the image from their compressed form. There are various kinds of noises like Gaussian noise, Passion noise, Spectral noise [4] etc. . These noises can be removed by applying denoise operation. However, removing noise from an image is a time consuming process. In addition, it is needed to apply appropriate denoising algorithm with proper arguments to remove the noise, in order to recover the original image. The noise model is given by

$$
I^{\prime}=D(I)+h
$$

Here, $\mathrm{D}(\mathrm{I})$ represent the degraded image by blurring operation w.r.t. tocleaned image $\mathrm{I}$. $\mathrm{h}$ is the Gaussian distributed additive noise.The new mindset of obtaining information from the images has lead to the use of a different way to obtain the underlying characteristics from the image [5]. Now-a-days CNN provides a ray of hope in removing these noises in a better fashion with promising results.

Since it was very difficult to obtain the original image from strong noise, we are using a different CNN architecture in deep learning model.

Three types of noise namely, shaky image, Gaussian noise and dark images are dealt in this paper for image correction/restoration. Effects of this noise are considered in this section. 
In this paper, we explored the a modified CNN U-Net [3]- [8] architecture for image denoising by deep learning model and developed our own localU-net model for denoising strong noise in the images. In Section II, we introduce literature and previous work. 3 kinds of noises we used as denoising targets. Section IIIprovides a brief U-Net deep learning model overview and explains comparative studies of the 2 models based on U-Net and modified U-net; Section IV and V describes training, experimental details and experiment results; Section VI concludes this work.

In our study, 3 kinds of noises: Gaussian noise, Blockiness and Camera shake are selected as the target for image correction. This section introduces these types of noises and their effects.

\section{PREVIOUS WORK}

In the last decades and few years, deep convolutional neural networks (DCNN) based data driven approaches showing exceptional results in several image processingtasks, such as image classification, segmentation, pixel to pixel encoding, and image super resolution [6], [7]. CNNs based features learning by convolutions operations are proposed for many inverse ill-posed issues including image denoising and image reconstruction [8]-[14].

Recently U-net decoder and encoder architecture has shown Exceptional results for image segmentation, image restorations tasks [15]-[18].The proposed work uses the local neighbors' data to obtain the statistics for reconstruction of noisy image. LM U-net model denoisethe degraded images instead of traditional denoising imaging techniques [5-8] such as spatial filtering, frequency domain approaches, wavelet, and few transform domain filtering. A good denoising model should remove high level as well as low level noise as much as possible along with preserving salient edges. Results obtained using CNN models proved better results than traditional techniques and are more computationally efficient

\section{Deep Learning Models}

Author [1], proposed, designed and implemented U-net DCNN model for image denoising using auto encoder architecture in Deep Learning model. The ambition of the work was to reconstruct close to original data which is free from Noise. Two main level architecture that were considered in this work are
A. U-Net Architecture.
B. Reformed U-Net

The designing details of the above said two architectures are explained as below.

\section{A. U-Net Architecture.}

In our analysis we are prudently taking a deep dive into U-Net architecture as base generator model. [2]-[5] is the U-net architecture on which we are inspired to delve on. Architecture model of the network is given below which formsan $U$ shaped network. The network basically encodes to compress and decodes on the rights hand side to reconstruct the denoisy image.

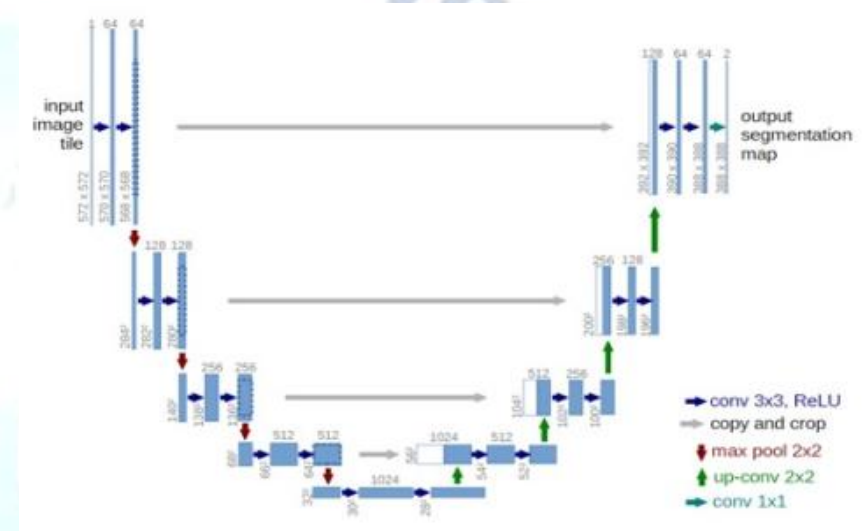

Figure 1 Architecture of U-Net [4]

This U-Net is not only used in image segmentation.Also used for image restorations applications.Segmentation helps to reduce the size of the image and capture the features. It compresses large size to few pixels of data by extracting features. The threshold can be used to reconstruct image base on global and local parameter features. Lost images can be restored using local feature extractions. When we apply convolution it creates a large number of parameters to train the model. Convolution layers reads the actual input of picture size, and give the output based on the number of neuron In that layers, kernel size, padding size if applied and stride the kernel that takes while convoluting. We can apply multiple layer of convolution so that model can learn better.

\section{B. LM U-NET}

DCGAN: Deep Conditional GANs [8], performance is enhanced by replacing pooling layers with fractional-stride convolutions and using Batch Normalization (Batch Normalization normalizes layer's input and helps higher learning rate [9] to each layer's output in model. Following these techniques, we reformed the LM U-Net shown in Figure 2. We named this proposed U-net "local 
modified U-Net". Figure 2 shows the architecture of local modified U-Net.We named this proposed U-net as " LM- U-Net

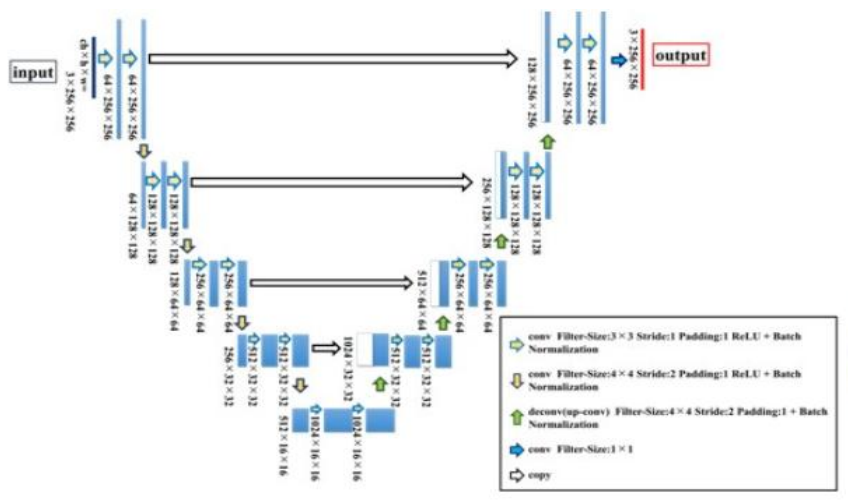

Figure 2 Architecture of LM-U-Net [2]

\section{TRAINING AND EXPERIMENTALDETAILS}

A high resolution image dataset is used for training. This image dataset is used in image segmentation process. Data set is split into train and test data set with 80: 20 rules and evaluating multiclass label issue.

\section{A. Programming:}

Our study involves Keras Deep learning framework. To build model here we are using Keras library. Noise is introduced using Gaussian filter and by thresholds. Goal is to produce images which are free from noise.

\section{RESULTS}

\section{A. Generation of Noisy Images}

Initially, all images, fortraining as well as testing were resized to $128 \times 128$ pixels. Gaussian distributed additive noise is then added to each low resolutions, resized image. Gaussian noise is generated by overlapping a clear image with Gaussian distribution whose parameter $\sigma$ is in the range $[15,50]$. Camera motion blur and shake is generated on averaging neighbor pixels by overlapping of the same images, each with a space-varying defocus work.

\section{B. Training and validation on train images}

Secondly, we have trained both models to generate clear, denoise images from noisy observations as shown in Figure 3. Training is carried-outon 500 epochs with mini batches of 15images per batch in step 1. In second step we have normalizednoisy image by dividing each pixel value by maximum value of the pixel.Step3. We calculated PSNR and SSIM between output and target image. Obtained PSNR values with state-of- the- art work and our proposed work is shown in table 1 .

\section{Evaluating of the trained Model}

To evaluate the trained model input noisy images and reconstructed denoise images are considered, we employed 2 different PSNR and SSIM estimate methods. Here we use PNSR: Peak signal-to-noise ratio which is the criterion for determining how close the output look like the target.

\section{Visualizing Output of The RGB Image}

Both the models proposed reduce the loss in training and testing process. Wehave evaluated clearly the model using the performance metrics. Got success in reducing noise from a given noisy image. We have compared our results with few state of the art works. Figure 4 and Figure 5 shows the visualized output result using the model trained. Using the PNSR and MAE criteria, both models could denoise. Also, the model trained over a larger number of epochs produced improved results than the one with a lesser number of epochs. As the number of layers in the network increase, we may end up in overfitting problems. Hence care is taken to obtain optimal value of systems training parameters
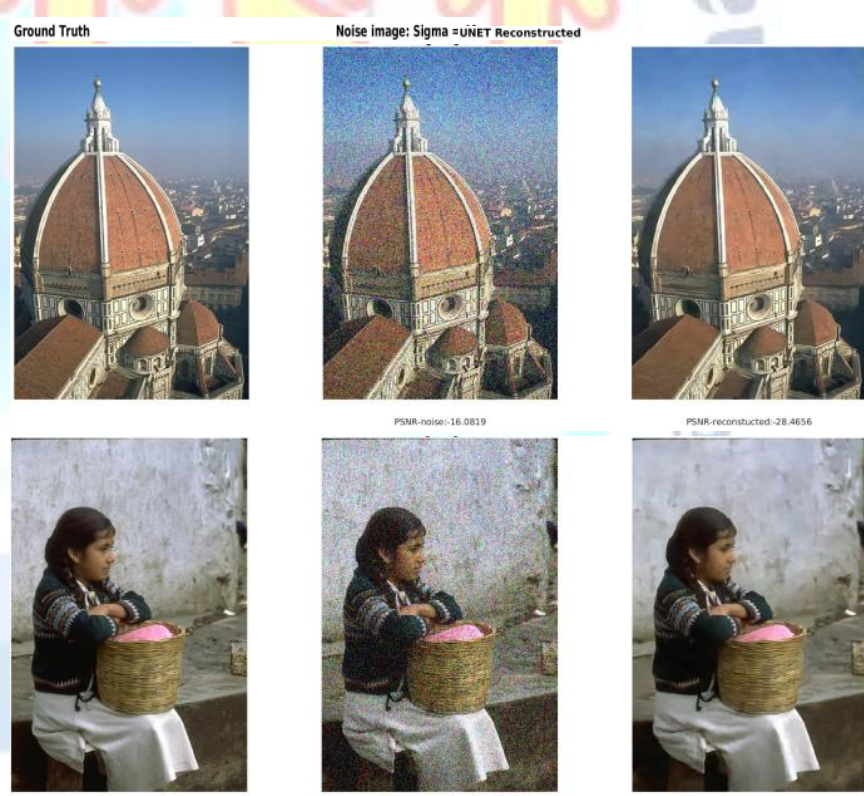

PSWR-nolise:-16 08:

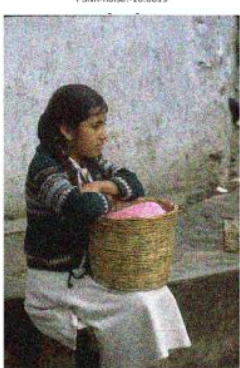

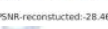
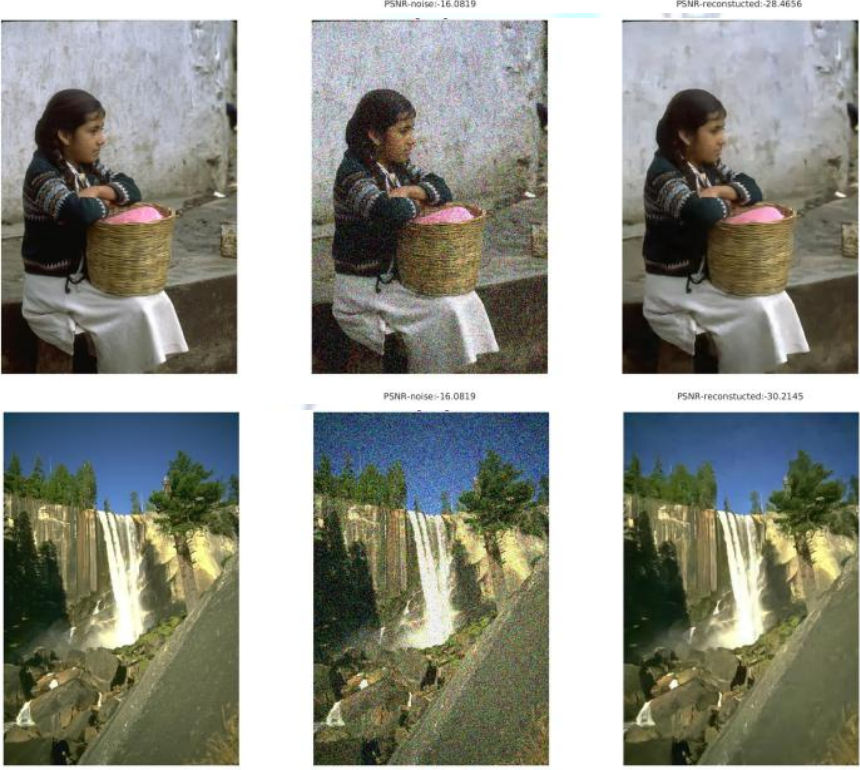

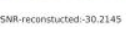

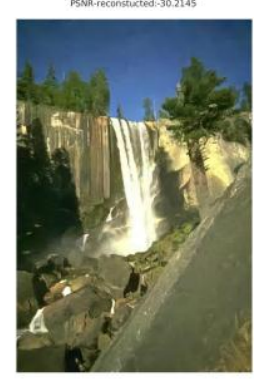

(b)

(c)

(a)

Figure 3. (a) clear image (b) Nosiy image with $\sigma$ parameter 40 . (c) denoisy image with our proposed LM U-net 

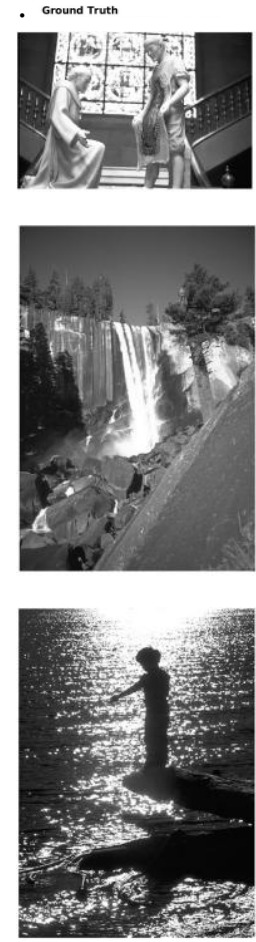

(a)
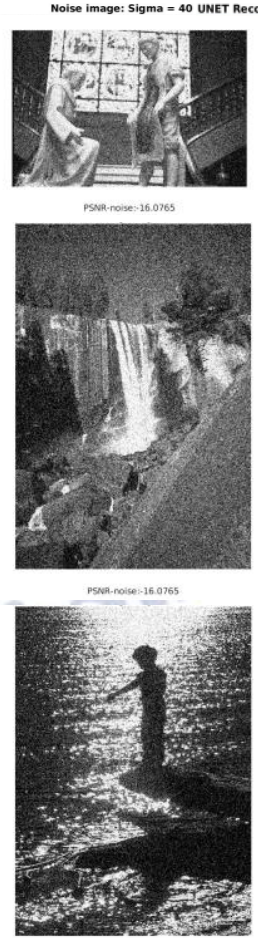

pseren.
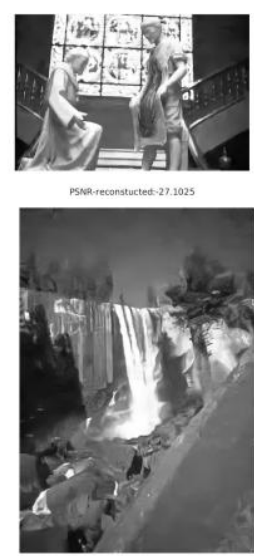

(1)

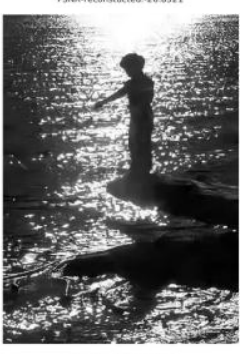

5.20.

(b) (c)

Figure 4. Gray images (a) clear image (b) Nosiy image with $\sigma$ parameter 40. (c) denoisy image with our proposed LM U-net
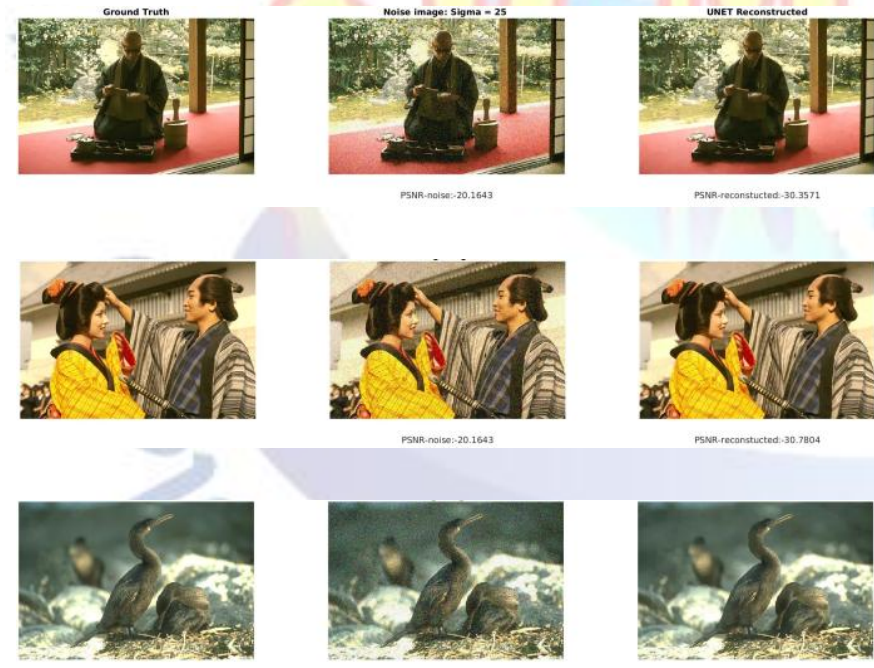

PSSRR:nolse: 20.1643
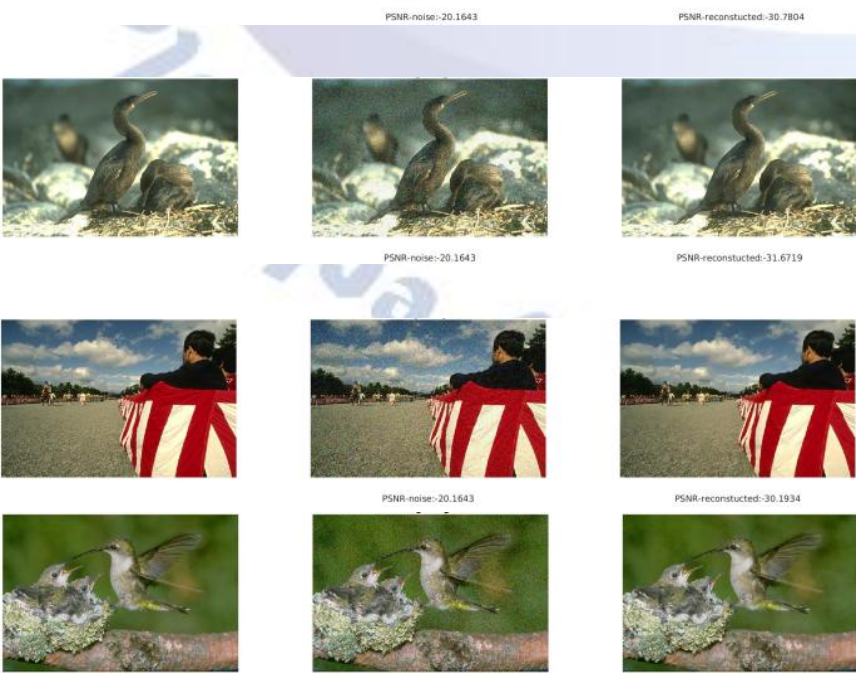

(a)

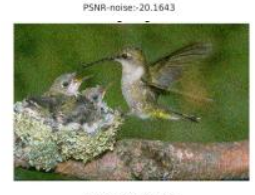

(b)

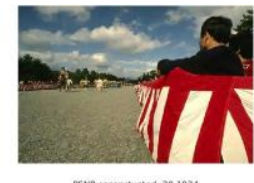

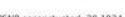

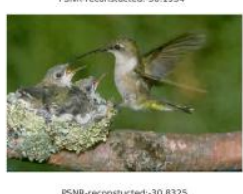

(c)

Figure 5. (a) clear image (b) Nosiy image with o parameter 40. (c) denoisy image with our proposed LM U-net

Table 1. Comparison result dealing with strong noise

\begin{tabular}{|c|c|c|c|}
\hline & $\begin{array}{c}\text { Gaussian } \\
\text { noise }\end{array}$ & $\begin{array}{c}\text { Salf and } \\
\text { pepper }\end{array}$ & $\begin{array}{c}\text { Camera } \\
\text { shake }\end{array}$ \\
\hline U-net & 0.9458 & 0.8637 & 0.8347 \\
\hline $\begin{array}{c}\text { LM } \\
\text { U-net }\end{array}$ & 0.9642 & 0.8745 & 0.84794 \\
\hline
\end{tabular}

\section{CONCLUSION}

Our experimental results shown on LM U-net Deep learning CNN are effective in denoising gray as well as RGB images. Further, we obtained both visual quality and quantity outputs compared with state-of-the-art work. The DCNN network model based on LM U-Net be successful in image denoising with the connections between decoding contract forward path and encoding path. The peak signal to noise ratio and SSIM calculated on ground truth and reconstructed gives better output. The calculated and measured loss values depending on each and every pixel. The prosed LM U-net can also be effectively utilized for many other image restoration task similar to image super resolution, image deblurring, image dehazing etc. Further study includes image dehaze and derain application

\section{REFERENCES}

[1] MasanaoKoeda, Takayuki Nakamura \& Etsuko Ueda, (2014) "Introduction to image Processing withOpenCV, 2nd ed", odansya, (Japanese).

[2] Pascal Vinent, Hugo Larochelle, YoshuaBengio\& Pierre-Antoine Manzagol, (2008) "Extracting and composing robust features with denoising autoencoders", Proceedings of the 25th international conference on Machine learning, ACM, pp1096-1103.

[3] Olaf Ronneberger, Philipp Fischer \& Thomas Brox, (2015) "U-net: Convolutional Networks for Biomedical Image Segmentation", International Conference on Medical image computing and computer-assisted intervention (MICCAI), Springer, Vol. 9351, pp234-241.

[4] Phillip Isola, Jun-Yan Zhu, Tinghui Zhou \& Alexei A. Efros, (2017) "Image-to-Image with Conditional Adversarial Networks", 2017 IEEE Conference on Computer Vision and Pattern Recognition (CVPR), pp5967-5976

[5] H N Latha, HN Poornima, "Estimation of Blur and Depth_Map of a De-focused Image by Sparsity using Gauss Markov Random Field Convex-Prior" International Journal of Science and Research, ISSN: 2319-7064, 2019

[6] Latha, H. N., Palachandra, M. V., \&Rao, M. Real Time Implementation and Performance Evaluation of WCDMA System over AWGN Channel on TMS320C6713DSK.Procedia Technology, 4, 82-86. 2012.

[7] J. Pan, Z. Hu, Z. Su, and M.-H. Yang, "LO-regularized intensity and gradient prior for deblurring text images and beyond," IEEE Proc. CVPR, 2016 
[8] Latha H N, Lakshmi M V and Ramachandran s “ Design Of Context Based Adaptive Variable Length Coding And Deblocking Filter for H. 264" Elsevier, Science Direct, PP 671676, 2012.

[9]Chakrabarti, T. Zickler, and W. T. Freeman, "Analyzing spatially-varying blur," in Computer Vision and Pattern Recognition (CVPR), 2010 IEEE Conference on, pp. 2512-2519, IEEE, 2010.

[10] Latha H N, Kranti K.P, and Sahay R R, "Simultaneous blur map estimation and deblurring of a single spacevariantly defocused image." 2017 27th National Conference on Communications (NCC).IEEE, 2017.

[11] E. Luo, S. H. Chan, and T. Q. Nguyen, "Adaptive image denoising by targeted databases," IEEE Transactions on Image Processing 24, 2167-2181, 2019.

[12] H N Latha, R Sadhan, D Sampreeth,"Image understanding: Semantic Segmentation of Graphics and Text using Faster-RCNN" Dec 2018 IEEE, International Conference on NEWS, 1, PP 82-87

[13] Sheeba S and Latha H N, " Design \& Implementation of Saliency Detection Model in H. 264 Standard" IJSR, pp14-2020, 2014

[14] Latha H N and H N Poornima 'Estimation of Blur and depth Map of a Defocused Image by Sparsity usingGauss Markov random Field Convex-Prior' International Journal for Science and Research, Vol08, Issue 11, pp 728-733, Nov2019

[15] BSD 500 dataset. (blur segmentation dataset) [16] Latha, H. N. , and BharathiLokesh. "Denoising andDeblurring by Gauss Markov Random Field :AnAlternating Minimization Convex Prior." IJSR : 2319-7064, Nov 2019. 8 (11), 6

[17] HS Meharwade, HN Latha, Design of high speed face detection and tracking by skin segmentation International Journal of Engineering Science and Research (IJESR), 1-6, 2014

[18] HN Latha, S Rudresh, D Sampreeth, S M Otageri,“ Image understanding: Semantic Segmentation of Graphics and Text using Faster- RCNN " International Conference on Networking, Embedded and wireless Systems(ICNEWS). 14-17, DEC 2018.
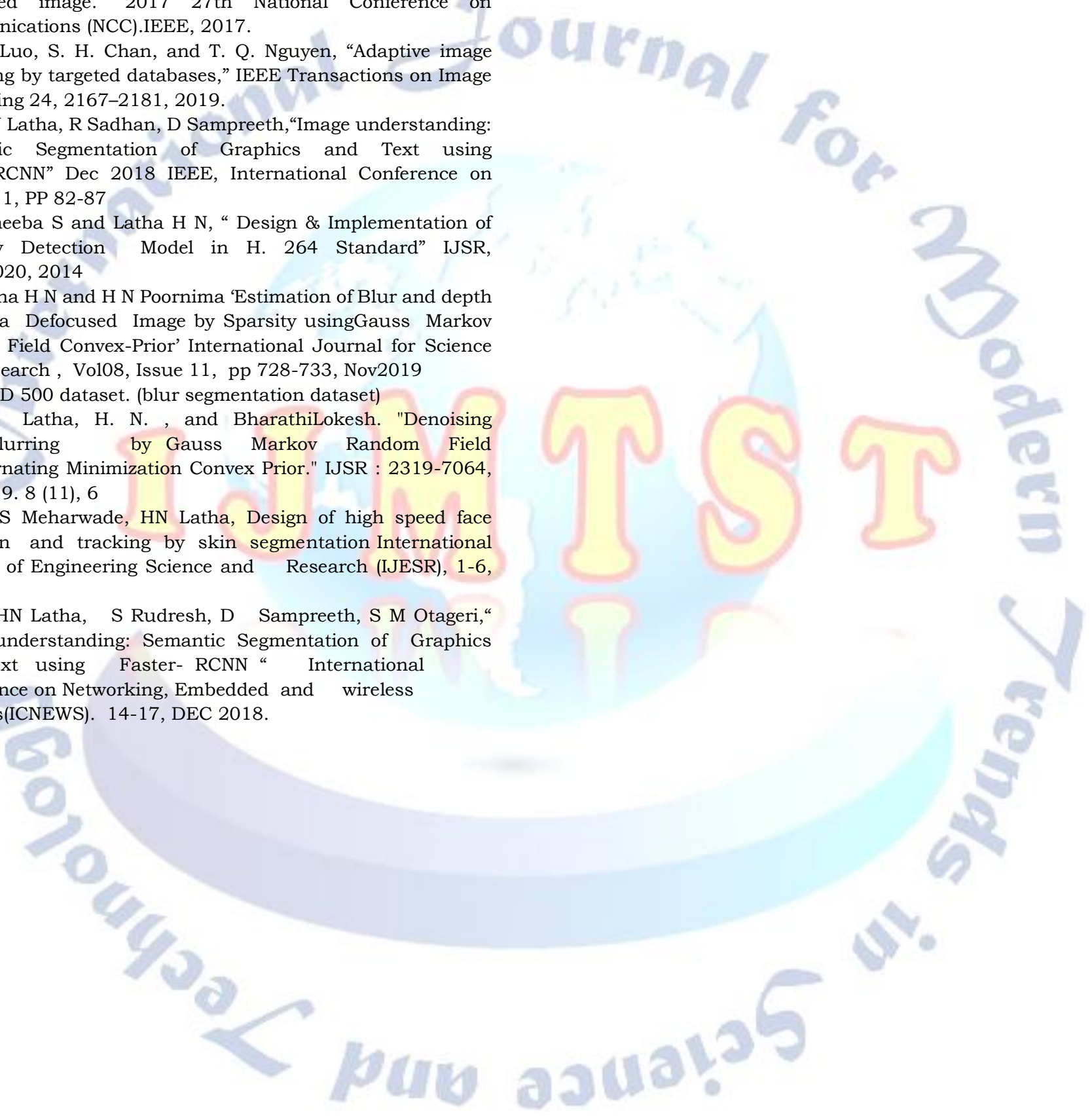